\title{
Genome-wide identification and expression profiling of DREB genes in Saccharum spontaneum
}

\author{
Zhen Li', Gang Wang ${ }^{2}$, Xihui Liu ${ }^{3}$, Zhengchao Wang ${ }^{4}$, Muqing Zhang ${ }^{5 *}$ and Jisen Zhang ${ }^{1,5^{*}}$
}

\begin{abstract}
Background: The dehydration-responsive element-binding proteins (DREBs) are important transcription factors that interact with a DRE/CRT (C-repeat) sequence and involve in response to multiple abiotic stresses in plants. Modern sugarcane are hybrids from the cross between Saccharum spontaneum and Saccharum officinarum, and the high sugar content is considered to the attribution of $S$. officinaurm, while the stress tolerance is attributed to $S$. spontaneum. To understand the molecular and evolutionary characterization and gene functions of the DREBs in sugarcane, based on the recent availability of the whole genome information, the present study performed a genome-wide in silico analysis of DREB genes and transcriptome analysis in the polyploidy S. spontaneum.

Results: Twelve DREB1 genes and six DREB2 genes were identified in S. spontaneum genome and all proteins contained a conserved AP2/ERF domain. Eleven SSDREB1 allele genes were assumed to be originated from tandem duplications, and two of them may be derived after the split of S. spontaneum and the proximal diploid species sorghum, suggesting tandem duplication contributed to the expansion of DREB1-type genes in sugarcane. Phylogenetic analysis revealed that one DREB2 gene was lost during the evolution of sugarcane. Expression profiling showed different SSDREB genes with variable expression levels in the different tissues, indicating seven $S S D R E B$ genes were likely involved in the development and photosynthesis of $S$. spontaneum. Furthermore, SsDREB1F, SSDREB1L, SsDREB2D, and SsDREB2F were up-regulated under drought and cold condition, suggesting that these four genes may be involved in both dehydration and cold response in sugarcane.

Conclusions: These findings demonstrated the important role of DREBs not only in the stress response, but also in the development and photosynthesis of S. spontaneum.
\end{abstract}

Keywords: Saccharum spontaneum, DREB, Phylogenetic analysis, Gene expression, Dehydration response

\section{Background}

Plants are exposed to various abiotic stresses such as drought, salinity, and extreme temperature, which cause adverse effects on their growth and yield [1]. A number

\footnotetext{
* Correspondence: zmuqing@163.com; zjisen@fafu.edu.cn

${ }^{5}$ Guangxi Key Laboratory of Sugarcane Biology, Guangxi University, Nanning 530004, Guangxi, China

${ }^{1}$ Fujian Provincial Key Laboratory of Haixia Applied Plant Systems Biology, College of Agriculture, Fujian Agriculture and Forestry University, Fuzhou 350002, China

Full list of author information is available at the end of the article
}

of genes are induced or repressed by these stresses to help plants to survive from these bad conditions, which can be divided into the gens coding stress tolerance proteins and the other coding regulatory proteins $[2,3]$. Transcription factors (TFs) are necessary for regulating the expression of stress-responsive genes. Dehydration responsive element binding proteins (DREBs) are the important TFs that regulate stress-responsive genes expression in the abscisic acid (ABA)-independent pathway [4]. DREBs belong to a subfamily of the APETALA2/ ethylene-responsive element-binding protein (AP2/

(c) The Author(s). 2021 Open Access This article is licensed under a Creative Commons Attribution 4.0 International License, which permits use, sharing, adaptation, distribution and reproduction in any medium or format, as long as you give appropriate credit to the original author(s) and the source, provide a link to the Creative Commons licence, and indicate if changes were made. The images or other third party material in this article are included in the article's Creative Commons licence, unless indicated otherwise in a credit line to the material. If material is not included in the article's Creative Commons licence and your intended use is not permitted by statutory regulation or exceeds the permitted use, you will need to obtain permission directly from the copyright holder. To view a copy of this licence, visit http://creativecommons.org/licenses/by/4.0/. The Creative Commons Public Domain Dedication waiver (http://creativecommons.org/publicdomain/zero/1.0/) applies to the data made available in this article, unless otherwise stated in a credit line to the data. 
ERFBP) superfamily of TFs, and can bind a dehydrationresponsive element (DRE) with the core motif A/ GCCGAC that was found in the promoter of many dehydration- and cold stress-inducible genes $[1,5]$. Each DREB protein contains a conserved AP2/ERF DNAbinding domain, which consist of $\sim 60$ amino acids $[6$, 7]. The three-dimensional structure of AP2/ERF domain revealed this domain comprises a three-strand antiparallel $\beta$-sheet and an $\alpha$-helix packed similarly parallel to the $\beta$-sheet [8]. Two amino acids, the 14th valine (V14) and 19th glutamic acid (E19) in the AP2/ERF domain of DREB proteins are conserved and play a central role in determining the DNA-binding specificity of DREB proteins [1]. On the basis of the similarities in the AP2/ERF domain, DREB subfamily has been divided into 6 subgroup (A-1 to A-6), and the canonical DREB proteins belong to subgroups A-1 (DREB1) and A-2 (DREB2) [1, 9].

Though DREB genes are mainly involved in the process regulating the drought stress, other functions have been noted for some DREB genes. Previous studies have demonstrated that $D R E B$ genes can be induced by various abiotic stresses, including drought [10-13], low temperatures [14-17], heat stress [18-20] and high salt [21-23]. Overexpressing OsDREB2A in soybean enhanced salt tolerance by accumulating osmolytes and improving the expression levels of some stressresponsive genes and TFs [24]. In transgenic Salvia miltiorrhiza, AtDREB1A and AtDREB1B both play a positive role in plant drought stress tolerance [12, 25]. $P v D R E B 1 C$ gene is transcriptionally down-regulated in response to salt stress, whereas $P \nu D R E B 1 C$ overexpression improves plant salt tolerance in transgenic tobacco. On the other hand, ectopic overexpression of PvDREB1C has been characterized as a negative regulator of cold stress response [16]. StDREB2 has been reported to play an important role in the drought stress tolerance of cotton (Gossypium barbadense L.) [26].

Sugarcane (Saccharum spp.) is a major crop mostly grown in tropical and subtropical regions worldwide, and adversely affected by drought, salinity, low temperature, high temperature, etc. Modern sugarcane cultivars are complex autopolyploid and aneuploidy of interspecific hybrids derived mainly from $S$. officinarum and S. spontaneum. For Saccharum hybrid, S. officinarum was assumed to contribute to genetic background of high sugar content, and S. spontaneum contributed to the stress tolerance and pest and disease resistance [27]. In China, over $70 \%$ of sugarcane were cultivated in the hilly area which contained a low level of soil water content during the drought season. Thus, enhancing drought tolerance has been an important target for improving the yield of sugarcane in field. According to previous researches, transgenic sugarcane transformed with
AtDREB2A CA showed the enhanced drought tolerance without biomass penalty [28]. Overexpression of EaDREB2 (Erianthus arundinaceus DREB2) in sugarcane enhances the drought and salinity tolerance, what's more, co-transformation of EaDREB2 and PDH45 (pea DNA helicase gene) shows lower drought tolerance but higher salinity tolerance than EaDREB2 alone [29]. Huang et al. recently had analyzed the DREB subfamily in S. spontaneum [30], here, we focused on the canonical $D R E B$ genes (DREB1s and DREB2s) and discriminated the genes and their alleles. We also explored the gene function based on large scales of expression profiles from RNA-seq data sets including leaf developmental gradient, diurnal cycle, development stage, drought stress and cold stress. Thus, this study may provide insights into the polyploid characterizes for the DREBs and function relative to photosynthesis and plant development beside the drought stress.

\section{Results}

Identification of SsDREB genes in S. spontaneum genome

A total of 277 proteins containing AP2/ERF domain(s) were originally obtained in the sugarcane $S$. spontaneum AP85-441 $(1 \mathrm{n}=4 \mathrm{x}=32)$ genome. Based on the classification of the AP2/ERF superfamily in Arabidopsis [1], 54 of them, containing multiple AP2/ERF domains, were classified into the AP2 subfamily. Thirteen of these proteins, possessing both AP2/ERF and B3 domains, were belonged to the RAV subfamily. Thirty-one proteins lacked a conserved WLG motif. Of the remaining 179 proteins, containing only one AP2/ERF domain with a conserved WLG motif, 83 were classified into the DREB subfamily (Group A) and 96 were classified into the ERF subfamily (Group B). Two canonical subgroups of DREBs (DREB1 and DREB2) were 20 and 10 proteins in S. spontaneum, respectively. After re-annotating manually with the assistance of FGENESH (http://www. softberry.com/berry.phtml? topic $=$ fgenesh\&group $=$ programs\&subgroup=gfind) [31], one protein in DREB1 subgroup was identified without AP2/ERF domain and deleted for further researching. Furthermore, these $D R E B$ genes have 1 to 4 alleles, including 1 gene with four alleles, 2 genes with three alleles, and 4 genes with two alleles (Additional File 1). Based on their chromosomal locations, we renamed these DREB1s and DREB2s as SsDREB1A to SsDREB1L, and SsDREB2A to $S s D R E B 2 F$, respectively, and additional -1 to -4 were added to the gene name for their alleles (Additional File 1).

Gene characteristics, including the length of protein sequences (AA), the molecular weight (MW), the theoretical isoelectric point (pI), the aliphatic index (AI), the grand average of hydropathicity (GRAVY), and the instability index (II) were analyzed (Additional File 1). The 
protein length were ranged from 186 to 390 aa, while the MW of the proteins from 20,362.44 Da to $41,745.7$ $\mathrm{Da}$, and the $\mathrm{pI}$ from 4.78 to 10.53 (Additional File 1).

\section{Multiple sequence alignment and phylogenetic analysis of SsDREBs}

All SsDREB protein sequences were found to have an AP2/ERF domain, with a highly conserved WLG motif (Additional File 2). Additionally, SsDREB2 proteins possessed a conserved 14th valine (V14) and a 19th glutamic acid (E19), whereas SsDREB1A to SsDREB1I did not have the glutamic acid in the E19 position (Additional File 2). In DREB1 subgroup, a nuclear localization signal (NLS) sequence 'P/KKR/KP/RA/TGRT/ KKFRETRHP' and a DSAW motif nestle up to the AP2/ ERF domain in the upstream and downstream, respectively. The LWSY motif was found at the end of the Cterminal region in most SsDREB1 proteins, except for SsDREB1A-1, SsDREB1A-3, SsDREB1F-2 and SsDREB1J (Additional File 2). In comparison with DREB1s, all DREB2 protein contained a CMIV-1 ([K/R]GKGGPxN) motif, and a PKK-like NLS sequence 'RKxPAKGSKKGCMxGKGGPENxx' was found at the upstream of AP2/ERF domain except SsDREB2E (Additional File 2).

In this study, we collected the DREB orthologous in Arabidopsis, rice, maize and sorghum (Table 1). It's worth noting that there are two more DREB1 genes and one less DREB2 genes in S. spontaneum than that in the proximal species sorghum. A phylogenetic tree of the SsDREB proteins and their orthologous was constructed (Fig. 1). Interestingly, the AtDREB proteins were clustered separately from the proteins which were derived from monocots in the DREB1-type genes, while clustered together with other proteins in the DREB2-type genes. A DREB2-type gene ABI4 belongs to the A-3 subgroup, and those identified in Arabidopsis, rice, maize and sorghum were formed a clade, but not found in $S$. spontaneum (Fig. 1), indicating that $A B I 4$ gene may be lost after the species divergence between $S$. spontaneum and sorghum.

Table 1 The number of DREB genes in Arabidopsis, maize, rice, sorghum, and S. spontaneum

\begin{tabular}{llll}
\hline Species & \multicolumn{2}{l}{ DREBs/CBFs } & Total \\
\cline { 2 - 3 } & DREB1 & DREB2 & \\
\hline A.thaliana & 6 & 9 & 15 \\
Z.mays & 10 & 10 & 20 \\
O.sativa & 10 & 6 & 16 \\
S.bicolor & 10 & 7 & 17 \\
S.spontaneum & $12(19)$ & $6(10)$ & $18(29)$ \\
\hline
\end{tabular}

The numbers in parenthesis detail the number of alleles of SsDREBs in S. spontaneum

\section{Location and duplication events among SsDREB genes}

The genome chromosome location information of SsDREBs showed that these 29 DREB alleles were unevenly distributed on the 14 chromosomes of $S$. spontaneum (Fig. 2a). Chromosome 2 (2A, 2B, 2C and 2D) contained the largest number of $S S D R E B$ genes, in addition to chromosome 7A with two SsDREB2 genes and other chromosomes only with one SsDREB2 gene (Fig. 2a).

Furthermore, according to the methods of Holub [32], a chromosomal region within $200 \mathrm{~kb}$ containing two or more genes is defined as a tandem duplication event. We identified 12 SsDREB1 allele genes (SsDREB1B-2/ $1 C-1 / 1 D$, SsDREB1A-3/1E, SsDREB1B-3/1G/1F-2/1H/ $1 A-4$, and SsDREB1C-2/1H), which were clustered into four tandem duplication event regions by BLASTP and MCScanx software, these tandemly duplicated regions were distrusted on the chromosome $2 \mathrm{~B}, 2 \mathrm{C}$ and $2 \mathrm{D}$ (Table 2). Chromosome 2D had two clusters, indicating a hot spot of $D R E B$ gene distribution. What's more, 17 $S s D R E B$ allele genes were results of the segmental duplication or whole-gnome duplication events, including all SsDREB2 genes (Additional File 3).

Among these tandemly duplicated gene pairs, SsDREB1C-2 and SsDREB1I, SsDREB1C-1 and SsDREB1D, possessed only one orthologous gene $S b D R E B 1 A$, while the orthologous $S b D R E B$ genes of SsDREB1A-3/1E and SsDREB1B-3/1G/1F-2/1H/1A-4 were also identified as tandemly duplicated gene pairs (Fig. 2b), indicating that tandem duplication events of SsDREB1C-2 and SsDREB1I, SsDREB1C-1 and SsDREB1D may happened after the divergence between S. spontaneum and sorghum. We therefore estimated the divergence time between tandemly distributed SsDREB genes and their orthologous SbDREBs based on the pairwise Ks (Table 3). The divergence time between S. spontaneum and its closest related diploid species sorghum had been estimated by Zhang et al. [33], it is 7.779 million years ago (Mya). In the current study, the divergence time between tandem-duplicated SsDREB1s and their orthologous SbDREB1s were ranged from 6.487 Mya to 18.874 Mya. In addition, the divergence time of SsDREB1C-2 and SsDREB1D with their orthologous were 6.487 Mya and 6.496 Mya, respectively, which are shorter than that of S. spontaneum and sorghum (7.779 Mya).

Gene structure and motif composition analysis of SsDREBs The exon-intron organizations and motifs of all SsDREB genes were examined in $S$. spontaneum. As shown in Fig. 3, all SsDREB genes had no intron except SsDREB1L, SsDREB2F and SsDREB2B with only one intron. The number and size of exon/intron among SsDREB alleles were highly conserved, while those in 


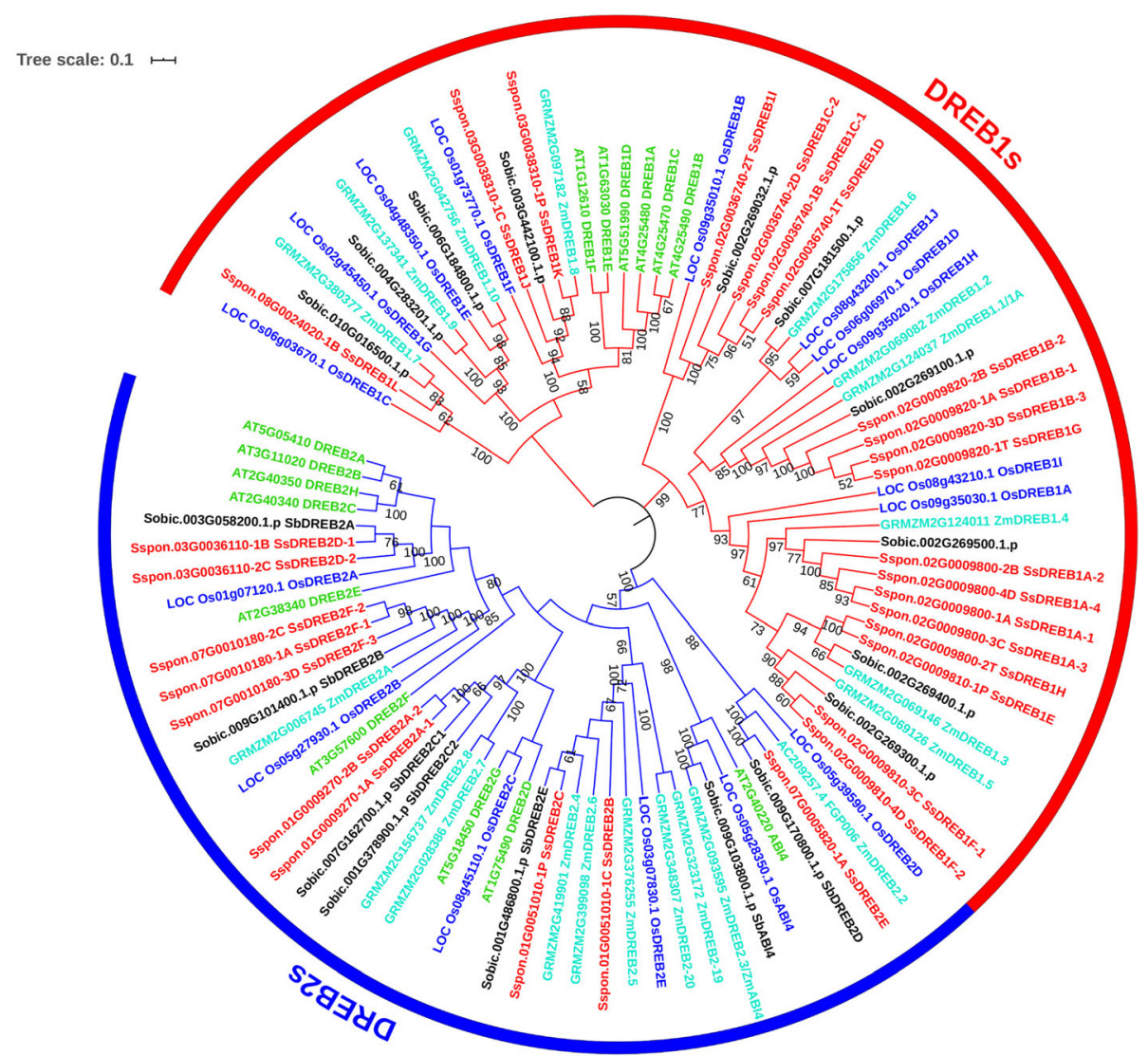

Fig. 1 Phylogenetic tree of DREB1 and DREB2 genes in S. spontaneum (red), sorghum (black), maize (cyan), rice (blue), and Arabidopsis (green). The phylogenetic tree was constructed based on the full-length sequence alignments of 97 DREB proteins from five species. Red and blue arcs indicate the DREB1-type and DREB2-type genes, respectively

SsDREB2F, SsDREB2F-2's intron were larger than other alleles. In addition, ten conserved motif sequences were detected (Fig. 3). All SsDREB genes contained Motif 1 and 2, which were related with AP2/ERF domain structure. Motif 3, 4 and 6 were only found in DREB1 genes, whereas Motif 7 was unique to DREB2 genes.

To identify the evolutionary forces acting on the SsDREB genes with alleles, the ratio of the nonsynonymous substitution rate to the synonymous substitution rate $(\mathrm{Ka} / \mathrm{Ks})$ was calculated. The $\mathrm{Ka} / \mathrm{Ks}$ ratios between SsDREB1A-3 and SsDREB1A-4, SsDREB2F-2 and SsDREB2F-3 were 1.401 and 2.450, respectively (Fig. 4), indicating that positive selection may be the dominant force driving the evolution of these two SsDREB genes.

\section{Expression analysis of SsDREB genes in S. spontaneum}

The expression patterns of SsDREB genes in different tissues and developmental stages of $S$. spontaneum were investigated by using transcriptomic data. The RNA-seq results of SsDREB1E, SsDREB1F, SsDREB1H and $S s D R E B 2 F$ were corroborated by real time quantitative reverse transcription-PCR (qRT-PCR) in three tissues (the first, 6th and 15th segments of 11-day-old second leaves) of S. spontaneum (Additional File 4). There is a significant positive relationship $\left(R^{2}=0.7491\right)$ between the relative expression level and the Fragments Per transcript Kilobase per Million fragments mapped (FPKM) value (Additional File 4), supporting the reliability of the gene expression based on RNA-seq.

Among the 18 SsDREB genes, 4 genes (SsDREB1I, SsDREB2A, SsDREB2B and SsDREB2C) were expressed at very low levels or undetectable in all examined tissues (Fig. 5). Transcripts of SsDREB2D was constitutively expressed in all these 12 tissues. The expression levels of SsDREB1E, SsDREB1F, SsDREB1H and SsDREB2F in leaves were higher than those in the stalks at different developmental stages. SsDREB1A exhibited much higher transcript levels in the leaves at maturing stage compared to other stages. The expression level of SsDREB1L increased with the maturity of the leaves, and gradually decreased from the top to bottom of the stem (Fig. 5). To further investigate the functions of DREB genes in the photosynthesis tissues of $S$. spontaneum. We exploited the continuously developmental gradient of 


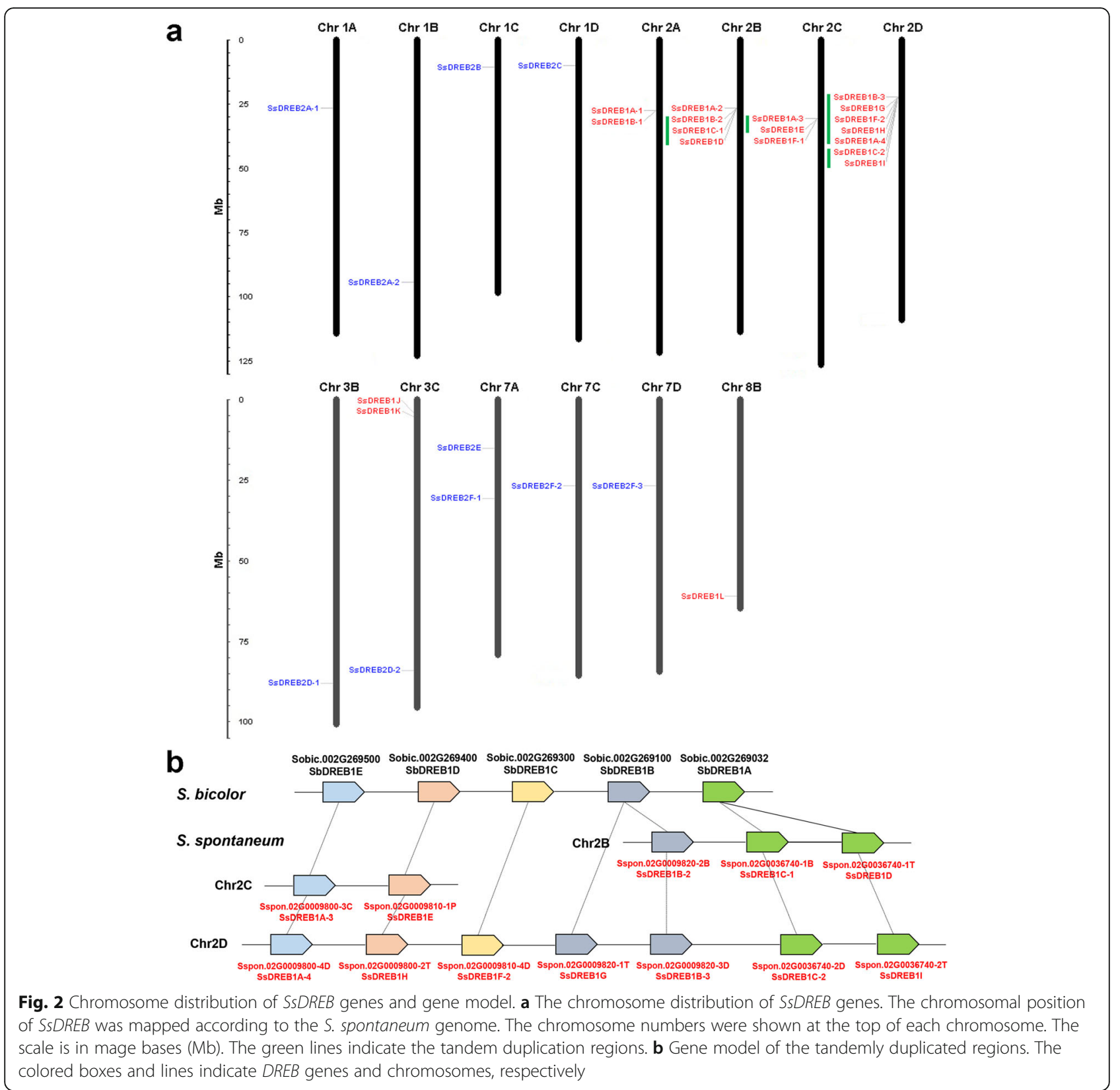

the leaf to analyze the transcriptome of SsDREBs. Similarly to the maize [34], the leaf of $S$. spontaneum can be divided into four zones, including a basal zone (base, 1 $\mathrm{cm}$ above the leaf two ligule, sink tissue), a transitional zone $(5 \mathrm{~cm}, 1 \mathrm{~cm}$ below the leaf one ligule, undergoing the sink-source transition), a maturing zone $(10 \mathrm{~cm}, 4$ $\mathrm{cm}$ above the leaf one ligule) and a mature zone (tip, 1 $\mathrm{cm}$ below the leaf two tip, fully differentiated and active C4 photosynthetic zones). Five genes (SsDREB1C, SsDREB1D, SsDREB1I, SsDREB2A and SsDREB2B) displayed undetectable or very low levels, suggesting that these genes play a very limited role in the developmental leaves in $S$. spontaneum. SsDREB1A, SsDREB1E,
SsDREB1F and SsDREB1H showed higher expression levels in mature zone than those in other zones of the leaf, whereas SSDREB1L displayed higher expression levels in the transitional zone, SsDREB1J and SsDREB1K showed higher transcript levels in the basal zone (Fig. 6). For the SsDREB2-type genes, SsDREB2F's transcript abundance gradually increased from the base to tip of the leaf, while the expression level of SsDREB2D gradually decreased from the base to tip of the leaf in $S$. spontaneum (Fig. 6). Additionally, we also collected samples for RNA-seq analysis at 2-h intervals over a 24-h period and 4-h intervals over an additional 24-h in $S$. spontaneum. SsDREB2F showed higher expression in the 
Table 2 Tandem duplication events in the SSDREB genes

\begin{tabular}{lllll}
\hline Cluster number & Gene name & Chromosome & Start site & End site \\
\hline 1 & SsDREB1C-2 & Chr2D & $22,208,944$ & $22,209,639$ \\
2 & SsDREB11 & Chr2D & $22,226,936$ & $22,227,607$ \\
& SsDREB1B-3 & Chr2D & $22,092,288$ & $22,093,103$ \\
& SsDREB1G & Chr2D & $22,107,221$ & $22,108,036$ \\
& SsDREB1F-2 & Chr2D & $22,113,187$ & $22,113,903$ \\
& SsDREB1H & Chr2D & $22,117,638$ & $22,118,345$ \\
& SsDREB1A-4 & Chr2D & $22,126,308$ & $22,127,015$ \\
& SsDREB1B-2 & Chr2B & $26,564,597$ & $26,565,409$ \\
& SsDREB1C-1 & Chr2B & $26,597,272$ & $26,598,093$ \\
& SsDREB1D & Chr2B & $26,612,975$ & $26,613,670$ \\
& SsDREB1A-3 & Chr2C & $30,543,015$ & $30,543,701$ \\
& SsDREB1E & Chr2C & $30,554,216$ & $30,554,923$ \\
\hline
\end{tabular}

light period than that in the dark period over these two 24-h cycles, indicating this gene may play an important role in diurnal rhythms (Fig. 7).

Furthermore, the transcriptome data of all SsDREB genes were analyzed in the primary meristem of the heart leaf in three drought-stressed sugarcane varieties. As illustrated in Fig. 8a, two SsDREB1 genes and two SsDREB2 genes were observed in response to drought stress, while the expression levels of SsDREB1A were slightly up-regulated after re-watering in three sugarcane varieties. SsDREB1F displayed similar expression patterns in these three sugarcane varieties, and its expression was gradually decreased with the increases of drought stress (Fig. 8b). What's more, the greatest drought-inducible gene was found in SsDREB1F under the mild drought stress. The expression of SsDREB1L was up-regulated by dehydration in the drought-tolerant F172, which was also induced by the mild drought stress in other two varieties. Interestingly, the transcript

Table 3 The divergence time between tandem-duplicated SsDREB genes and their orthologous SbDREBS

\begin{tabular}{lll}
\hline Gene pairs & Ks & Divergence time (Mya) \\
\hline SbDREB1A-SsDREB1C-2 & 0.079 & 6.487 \\
SbDREB1A-SsDREB1D & 0.079 & 6.496 \\
SbDREB1A-SsDREB1I & 0.096 & 7.841 \\
SbDREB1D-SsDREB1E & 0.133 & 10.917 \\
SbDREB1D-SsDREB1H & 0.140 & 11.496 \\
SbDREB1A-SsDREB1C-1 & 0.170 & 13.902 \\
SbDREB1B-SSDREB1B-2 & 0.180 & 14.747 \\
SbDREB1B-SSDREB1B-1 & 0.183 & 15.038 \\
SbDREB1B-SsDREB1G & 0.191 & 15.686 \\
SbDREB1E-SSDREB1A-4 & 0.213 & 17.444 \\
SbDREB1E-SSDREB1A-2 & 0.230 & 18.874 \\
\hline
\end{tabular}

abundances of SsDREB1L was increased slightly after rewatering under the moderate and severe drought stress conditions in GT31. Two SsDREB2 genes, SsDREB2D and $S S D R E B 2 F$, showed similar expression patterns with high expression levels. In contrast to the expressions under the normal growing conditions, the expressions of these two genes were up-regulated in response to dehydration, and then decreased after re-watering in all sugarcane varieties (Fig. 8b).

Finally, in order to investigate the response of SsDREB genes in cold stress, we analyzed the transcriptome expression profiles of all these genes in S. spontaneum under cold stress. Six SSDREB genes were induced by cold stress in hyperploid clone $15-28(2 \mathrm{n}=92)$ of $S$. spontaneum, and eight SSDREB genes were up-regulated in hypoploid clone $12-23(2 \mathrm{n}=54)$ (Fig. 8a). The greatest cold-inducible response was observed in SsDREB1F, whose expression was up-regulated more than 200-fold both in clone 15-28 and clone 12-23, in compare with expression under normal growing conditions. The induction response of SsDREB1A, SsDREB1B, SsDREB1E and $S S D R E B 1 F$ in clone 15-28 were higher than that in clone 12-23, while the expression levels of SsDREB1L and $S S D R E B 2 F$ in clone 12-23 were higher than that in clone 15-28 under cold stress. The expression of SsDREB1H and SsDREB2D were only up-regulated in response to cold stress in clone 12-23.

For the genes in tandemly duplicated regions, SSDREB1F-2 and SsDREB1H showed higher expression levels in leaves than those in stalks at different developmental stages, moreover, the expression levels of SsDREB1F-2, SsDREB1H and SsDREB1A-4 gradually increased from the base to tip of the leaf, whereas SsDREB1B-3 and SsDREB1G displayed a lower levels in all tissues (Additional File 5). In addition, the expression of $S S D R E B 1 F-2$ was significantly up-regulated in response to dehydration in three sugarcane varieties, while other SSDREB1 genes in tandemly duplicated clusters were expressed at very low levels or undetectable (Additional File 5).

\section{Discussion}

The DREB-type transcription factors have been recently identified in many plants, for instance, Arabidopsis [1], Brassica rapa [36], rice [37, 38], barley [39], sorghum [40], and maize [9]. DREB genes also play a key role in plant response to multiple abiotic stresses [41]. Thus, it's understandable that $D R E B$ genes may contribute to the enhanced stress tolerance and the improved production of sugarcane in field. However, the DREB genes have not been systematically studied in sugarcane because of its complex genetic background. In this study, 18 typical $D R E B$ genes in the S. sponteaneum genome were identified and analyzed using a bioinformatics approach to 

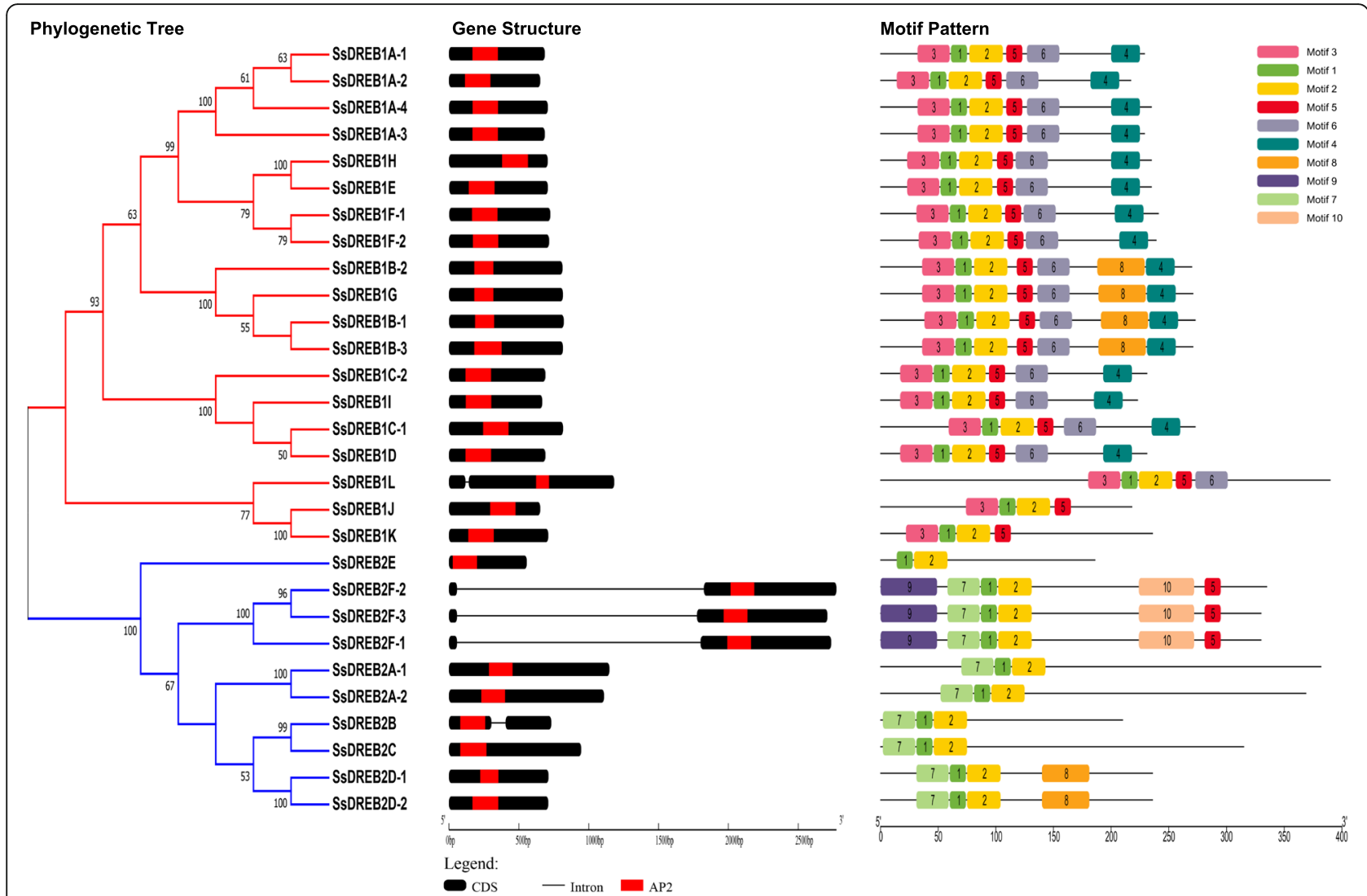

Fig. 3 Phylogenetic relationships, gene structures and conserved protein motifs for the SsDREB genes. The phylogenetic tree was constructed based one the full-length protein sequences of 29 SSDREB alleles using MEGA 7.0. Exons and introns are represented by black boxes and lines, respectively. The AP2 domains are highlighted by red boxes. The numbers 1-10 of motifs are displayed in different color boxes

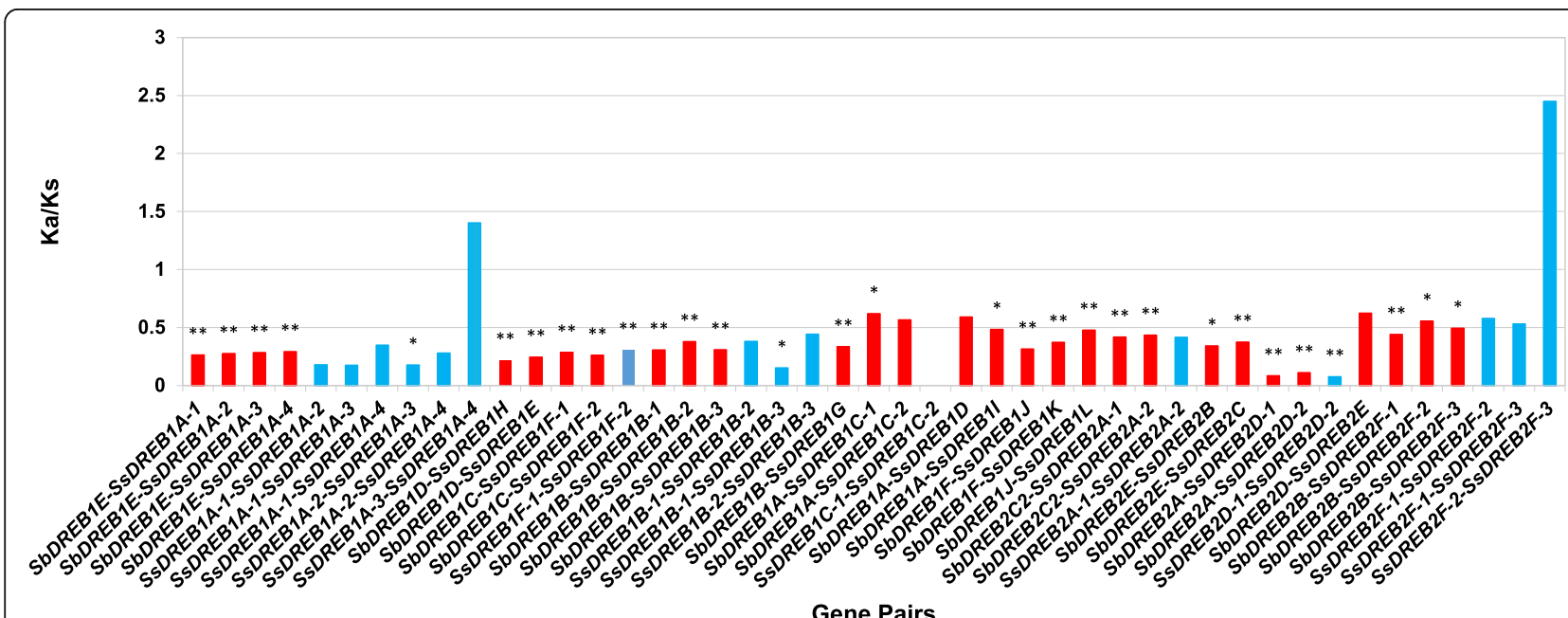

Fig. 4 The Ka/Ks of SsDREB alleles and SsDREB-SbDREB. The blue boxes indicate the Ka/Ks of SsDREB allele genes, the red boxes indicate the Ka/Ks of orthologous between sorghum and S. spontaneum. The $p$-value $<0.05$ is indicated by *. The $p$-value $<0.01$ is indicated by ** 


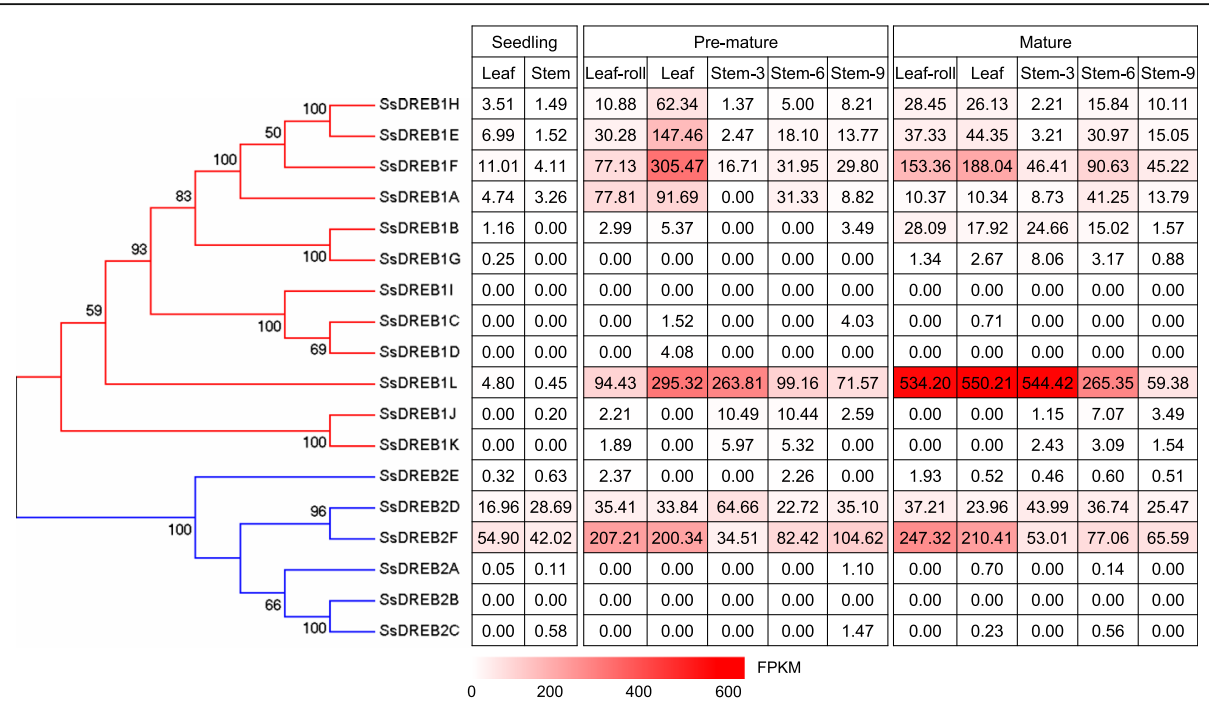

Fig. 5 The expression pattern of SSDREBS based on FPKM in different tissues of different stages in S. spontaneum

provide the clues for further functional investigations of SsDREB genes.

In the present study, 12 SsDREB1 genes and six SsDREB2 genes were identified in the S. sponteaneum genome, respectively. As a proximal species of sugarcane, there are ten DREB1-type genes and seven DREB2- type genes in the sorghum genome (Table 1). Phylogenetic analysis showed that a DREB2-type gene, $A B I 4$, lost the orthologous gene in $S$. sponteaneum, which explains the reason why $S$. sponteaneum have one less DREB2 genes, relative to the number of DREB2 gene in sorghum. Previous research has reported that DREB1 family

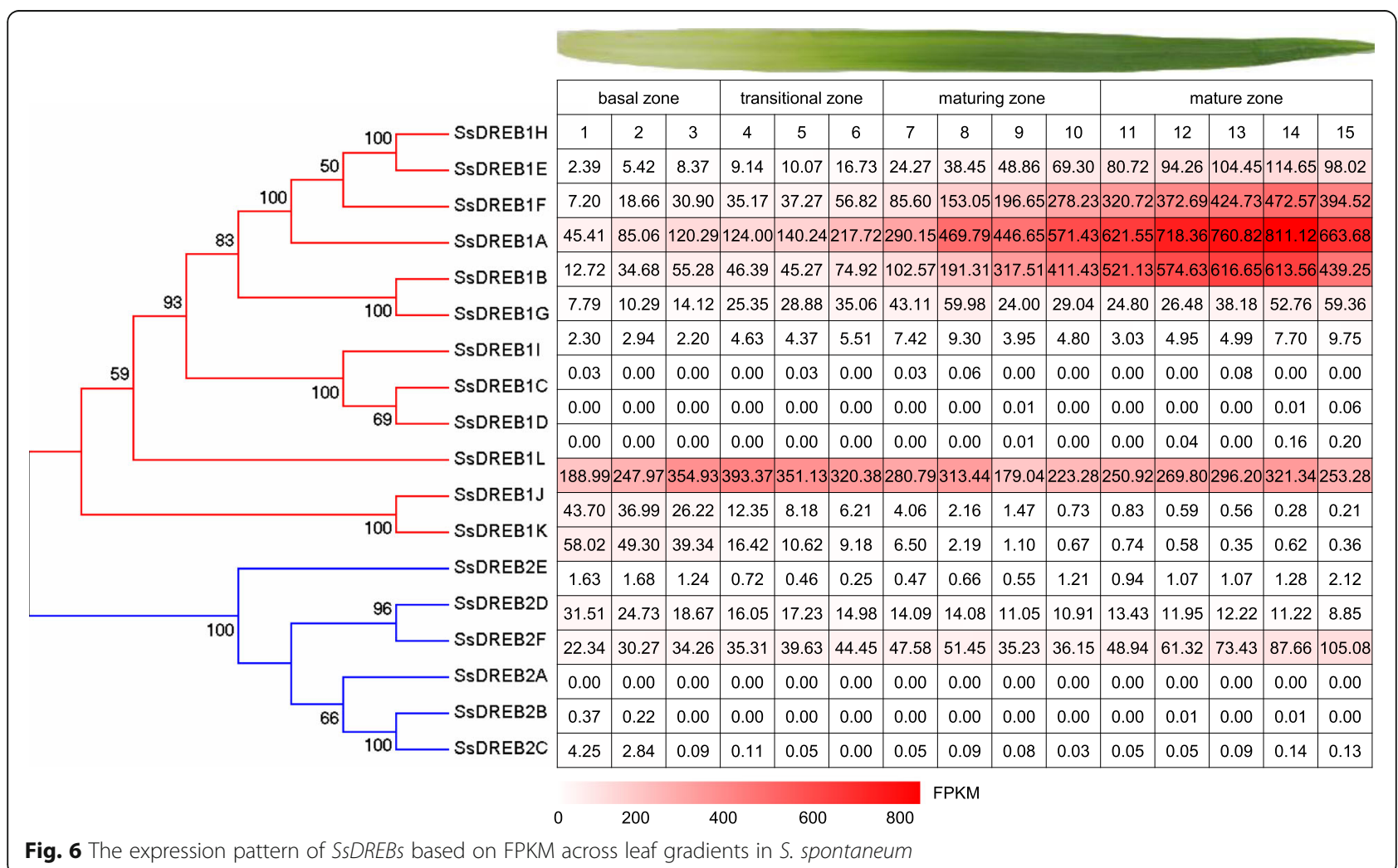




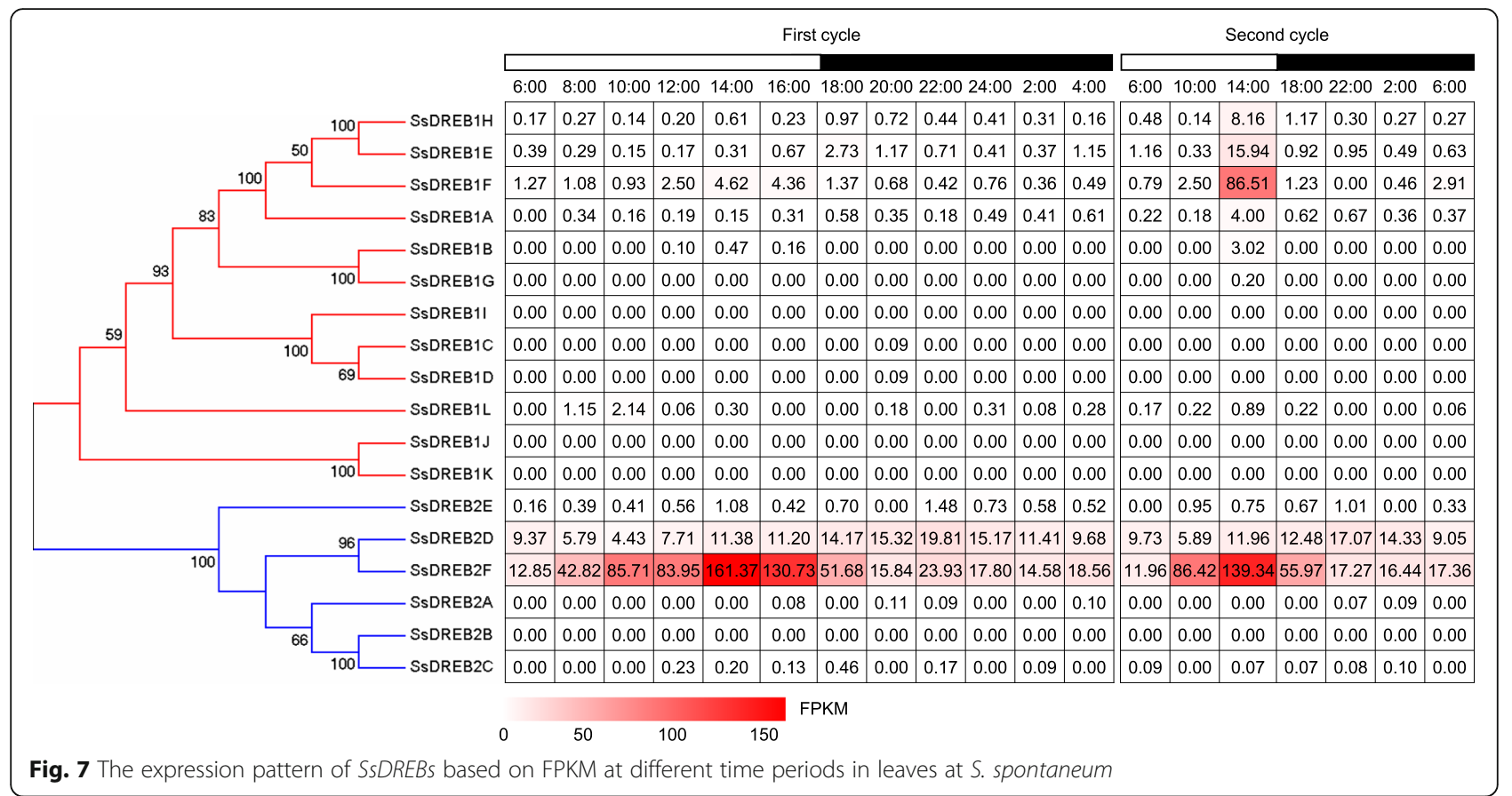

were expanded by the process of gene duplications [38]. Eleven tandemly duplicated SsDREB1 allele genes were found in this paper, among them, the divergence times of SsDREB1C-2 and SsDREB1D with their orthologous $S B D R E B$ are shorter than that of S. spontaneum and sorghum, suggesting that these two genes originated from tandem duplication after species differentiation between S. spontaneum and sorghum. Although all SsDREB2 genes were derived from segmental duplication or whole-gnome duplication events, no gene expansion has occurred compared with sorghum. That is tandem duplication events, instead of segmental duplication or whole-gnome duplication events contributed to the expansion of DREB genes in S. spontaneum. Previous studies have been reported that duplications resulting in some gene families represented functional redundancy and/or divergence [42-44]. In this study, of the SsDREB1 genes in Cluster 2 tandemly duplicated gene pairs, SsDREB1F-2 and SsDREB1H have the same expression pattern at different developmental stages and leaf gradient segments (Additional File 5), whereas SsDREB1B-3 and SsDREB1G expressed at very low levels, suggesting these tandemly duplicated genes may exist functional redundancy.

The V14 and E19 in the AP2/ERF domain are highly conserved and play a major role in the recognition and binding specificity of DRE cis-acting element [1]. However, some studies has reported that E19 is not conserved in DREB1 proteins in rice, wheat, barley and rye [41, 45]. Moreover, Dubouzet et al. reported that OsDREB1A do not has the glutamic acid in the E19 position, but binds specifically to the DRE element in the promoter of $r d 29 \mathrm{~A}$ genes [46]. These results indicate that V14 may be more important than E19 for the recognition of DNA-binding sequence in DREB1 proteins. That is, deletion of this glutamic acid in the E19 position of SsDREB1A-SsDREB1I genes (Additional File 2) has little effect on their functions. TFs only function in the nucleus, the regulation of their entry into nucleus is significant for their function. The NLS medicates the entry of TFs, and the TFs without NLS enters the nucleus by the interaction with the TFs with NLS [45]. The DREB1-type transcription factors are distinctly different from other subgroup DREBs, they have a NLS sequence 'PKK/RPAGRxKFxETRHP' at the upstream of AP2/ERF domain, while DREB2 proteins possess a PKK-like NLS sequence 'RKxPAKGSKKGCMxGKGGPENxx' immediately upstream of the AP2/ERF domain $[47,48]$. In the current study, all SsDREB1 proteins had a NLS sequence 'P/KKR/KP/RA/TGRxKFRETRHP', and most SsDREB2 proteins possessed a PKK-like NLS, except for SsDREB2E (Additional File 2), indicating that SsDREB2E might function differently from other SsDREB2 genes.

DREBs are considered as the master regulators of various abiotic stress responses, and also involved in the developmental processes of plants. For instance, OsDREB2A, OsDREB2B, and OsAIB4 were reported to involve in the embryo and endosperm development in rice [49]. In Arabidopsis, ABI4 was involved in the seed development and the lateral root formation [50, 51]. Given gene expression patterns are highly corrected with their functions in plants [52], SsDREB1E, SsDREB1F, 


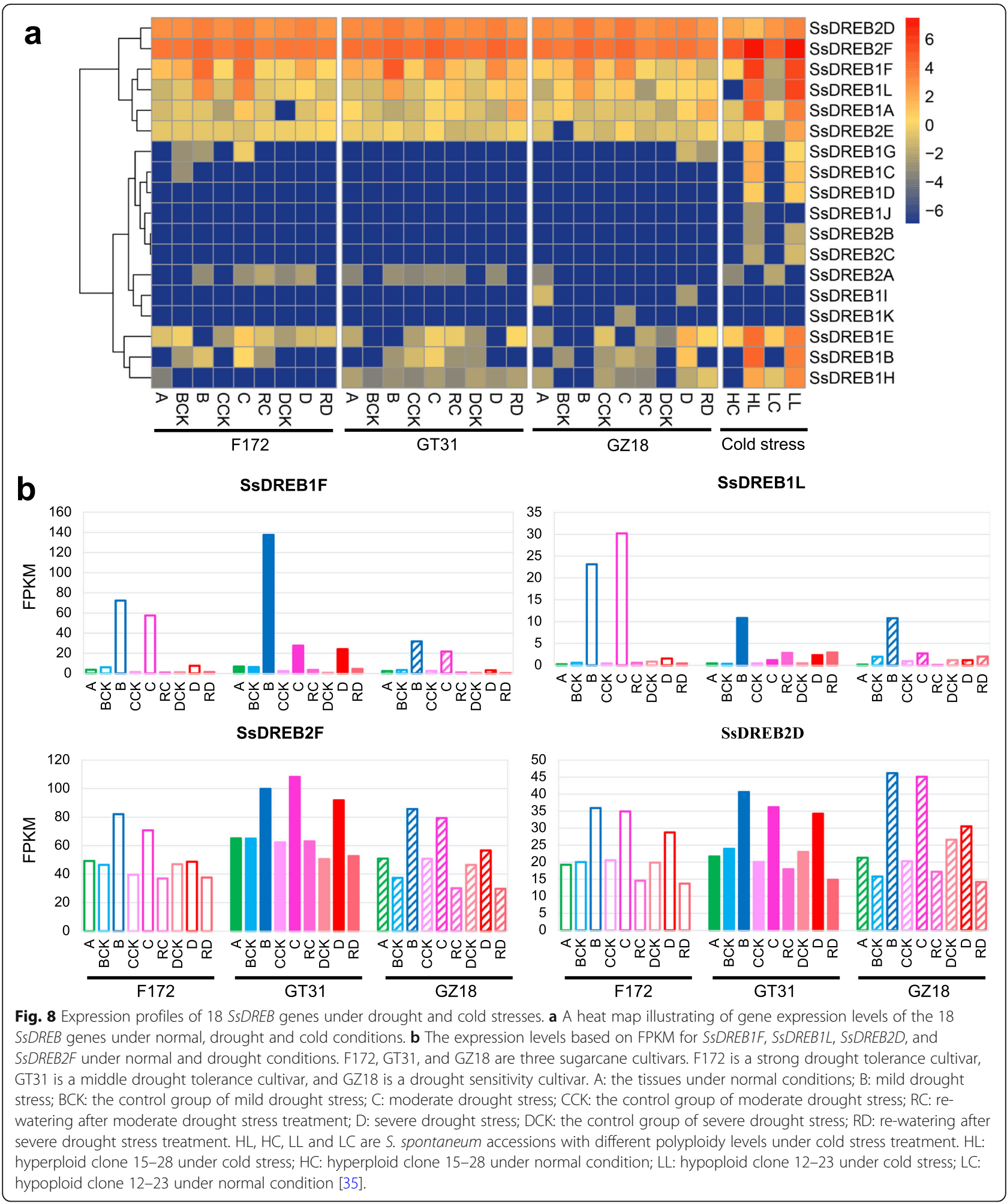

SsDREB1H, and SsDREB2F displayed higher expression levels in leaves than culms at different developmental stages of S. spontaneum in the present study (Fig. 5), suggesting these four genes might play a major role in the leaf development throughout the plant life cycle, while SsDREB1A showed related to the leaf development only at maturing stage. And the gradually increased expression pattern from the near-terrestrial end to the distal of steam at the stage of sugar accumulating suggested that SsDREB1L may play role relative to sugar 
metabolism. Constitutively expression of SsDREB2D in all tissues indicated that this gene may has a central role throughout S. spontaneum life cycle.

$\mathrm{Li}$ et al. reported that in the region of transition from the sink to source tissues of the leaf, the transcript abundance of genes associated with the photosynthetic machinery is increased [34], SsDREB1L exhibited higher expression levels in this transitional zone than other regions (Fig. 6), suggesting that this gene could be associated with the photosynthetic machinery of $S$. spontaneum. The peak expression levels of SsDREB1J, SsDREB1K and SsDREB2D in the undifferentiated basal region suggested that these three gene may regulate the basic cellular activities. The distal region of the leaf was fully differentiated with highest levels of photosynthesis, thus, the dominant expression levels of SsDREB1A, SsDREB1E, SsDREB1F, SsDREB1H and SsDREB2F in the distal region of the leaf indicated that these five genes may be highly correlated with the photosynthetic reactions in leaves. Previous researches have documented that the expression levels of some plant genes are effected by circadian rhythm, which gives plants the innate ability to measure time, and allows them to anticipate daily changes in the environment and to coordinate the developmental and metabolic processes induced by the environmental factors [53-58]. However, only SsDREB2F showed varied expression in the mature leaf during the diurnal cycle (Fig. 7), indicating that this gene might regulate the photosynthesis in mature sugarcane.

Previous efforts have been made to demonstrate that $D R E B 1 s$ or DREB2s involve the process regulating the stress in a number of plants, including maize, rice, cotton, and S. miltiorrhiza $[19,25,26,46,59]$. In this work, expression profile analysis revealed that SSDREB1F, SsDREB1L, SsDREB2D, and SsDREB2F were induced by drought stress in three sugarcane varieties (Fig. 8), suggesting these four $D R E B$ genes might play a key role in response to dehydration in sugarcane. The expression level of SsDREB1L was increased after re-watering in GT31, a sugarcane variety which is not drought-tolerant with the good recovering ability. This result indicated that this gene might help plant recover from drought stress in sugarcane. In addition, the expression levels of SsDREB1A, SsDREB1B, SsDREB1E, SsDREB1F, SsDREB1H, SsDREB1L, SsDREB2D and SsDREB2F were up-regulated under cold stress in S. spontaneum, indicating that these eight genes may be involved in responding to cold stress in S. spontaneum.

\section{Conclusions}

In summary, the present study identified 12 DREB1 genes and 6 DREB2 genes from S. spontaneum, and renamed them as SsDREB1A-SsDREB1L, and SsDREB2A-SsDREB2F on the basis of their chromosomal locations. Phylogenetic analysis based on the orthologous from sorghum, rice, maize, and Arabidopsis revealed that a DREB2-type gene, $A B I 4$, was lost during the evolution of $S$. spontaneum. Analysis of gene duplication showed that tandem duplication events contributed to the expansion of DREB1-type gene in $S$. spontaneum. In addition, these genes showed functional role in the sugarcane growth and development, photosynthesis, dehydration and cold stress response. However, how these $D R E B$ genes participate in the processes of development, and stress response remains to be further elucidated. Our present findings offer a useful information to understand the physiological functions of SsDREBs in sugarcane.

\section{Materials and methods \\ Plant materials}

The founding Saccharum species, S. spontaneum SES208 (originated in USA) was used in this paper [60]. The plant material was identified by Irvine JE [61], and the Saccharum species was planted in the campus of Fujian Agricultural and Forestry University (Fuzhou, China). The collection of S. spontaneum and the performance of experimental research on such plant were complied with the national guidelines of China.

The tissues of S. spontaneum at different developmental stages were obtained from leaf roll, leaf, top immature internode (Stem-3), premature internode (Stem-9) and mature internode (Stem-15) as previously described [62-64]. For leaf gradient experiment, S. spontaneum plants were grown under the following conditions: light intensity of $350 \mu \mathrm{mol} / \mathrm{m}^{2} / \mathrm{s}, 14: 10 \mathrm{~L} / \mathrm{D}, 30^{\circ} \mathrm{C} \mathrm{L} / 22^{\circ} \mathrm{C} \mathrm{D}$ and $60 \%$ relative humidity. 11-day-old second leaves were collected after planting $3 \mathrm{~h}$ into the light period and were cut into $151-\mathrm{cm}$ segments followed the approach described by Li et al. [34]. The mature leaves of S. spontaneum for the diurnal cycle experiment were collected at 12 time points $(6$ a.m., 8 a.m., 10 a.m., noon, 2 p.m., 4 p.m., 6 p.m., 8 p.m., 10 p.m., midnight, 2 a.m., 4 a.m.) and 7 time points ( 6 a.m., 10 a.m., 2 p.m., 6 p.m., 10 p.m., 2 a.m., 6 a.m.) from March 2 to 3, 2017 [64, 65].

The transcriptome data of sugarcane with drought treatment were obtained from Sugarcane Research Institute of Guangxi Academy of Agricultural Sciences (Nanning, China). We collected the first leaves of three 7month-old sugarcane cultivars (F172, strong drought tolerance; GT31, middle drought tolerance; GZ18, drought sensitivity) under normal conditions, mild drought stress, moderate drought stress, severe drought stress and re-watering for RNA-seq library construction. The transcriptome data of S. spontaneum under cold stress were obtained from Yang et al [35]. 


\section{Identifications of SsDREB genes}

A Hidden Markov Model (HMM) profile of the AP2/ ERF domain (PF00847) was obtained from the Pfam protein family database (http://pfam.xfam.org/) [66] and used to identify the proteins which contain AP2/ERF domain(s). Then we obtained DREB genes based on the similarities of the amino acid sequence and the number of AP2/ERF domains. Finally, the physical and chemical properties including AA, MW, theoretical pI, GRAVY, AI, and II of putative SsDREB proteins were calculated by the online ExPASy-ProtParam tool (http://web. expasy.org/protparam/). Manual annotation was performed for the genes that were incorrectly predicted. Additionally, DREB orthologous genes from sorghum, rice, maize, and Arabidopsis were collected [9, 37, 38, $40,67]$.

\section{Sequence analysis}

The AP2/ERF domain sequences of identified 29 SsDREB proteins were included in multiple sequence alignments using DNAMAN with default parameters. The exon-intron organization of SsDREB genes was determined based on their coding sequence alignments and respective genomics sequences using the online program Gene Structure Display Server (GSDS: http://gsds. gao-lab.org/) [68]. Conserved motif in SsDREB proteins were predicted using TBtools software with number of motifs to find: 10 and minimum-maximum width to find: 6-50 [69]. The non-synonymous (Ka) and synonymous (Ks) substitution ratios were calculated by the easy_KaKs calculation program [70]. The divergence time $(\mathrm{T})$ was calculated by $\mathrm{T}=\mathrm{Ks} /\left(2 \times 6.1 \times 10^{-9}\right) \times$ $10^{-6}$ Mya [71].

\section{Phylogenetic analysis}

The sequences of DREB proteins were aligned using MUSCLE in MEGA (version 7.0) with default parameters [72]. A phylogenetic tree based on the alignment was constructed using MEGA (version 7.0) with the neighbor-joining (NJ) method with the bootstrap test replicated 1000 times, the Poisson model, and Pairwise deletion $[73,74]$. The result was imported into the Interactive Tree Of Life (iTOL) program to create the phylogenetic tree [75].

\section{Chromosomal distribution and gene duplication}

The physical location of SsDREBs on the chromosomes was obtained from the database of $S$. spontaneum genome. MapInspect software (http://www.softsea.com/ download/MapInspect.html) was employed to visualize the chromosomal distribution of deduced SsDREB genes according to their initial position and length of chromosome. To analyze the duplication pattern for each SsDREB gene, the BLASTP program (E-value $<10^{-5}$ ) and Multiple Collinearity Scan toolkit (MCScanX) were used [76].

\section{Expression profiling analysis of DREBs in S. spontaneum based on RNA-seq}

HiSeq $^{\text {min }} 2500$ platform (Illumina Inc., CA, USA) by the Novogene Bioinformatics Technology Co., Ltd. (Beijing, China). The reads obtained from the sequencing instruments under drought and cold stress were filtered to remove adapters and low-quality reads by Trimomatic [77]. The reference genome of S. spontaneum AP85-441 (v20180123) is constructed to be indexed for further analysis by the align_and_estimate_abundance.pl of Trinity (version 2.8.5) [78]. Transcript expression levels of individual genes were quantified using FPKM values (fragments per kilobase of exon per million fragments mapped) by align_and_estimate_abundance.pl in Trinity [78] and value of the gene was calculated using the RNA-Seq by the Expectation-Maximization (RSEM) method.

Experimental validation of DREB gene expression level by qRT-PCR

RNA of each sample was in reverse transcription with the StarScript II First-strand cDNA Synthesis Mix with gDNA Remover (GenStar, A224-10) following the manufacturer's instructions. The qRT-PCR amplification was carried out using $2 \times$ RealStar Green Fast Mixture (GenStar, A301-10) on a Multicolor Real-Time PCR Detection System (Bio-Rad) and the reaction program was refer to the two steps method of the protocol from this kit: $95^{\circ} \mathrm{C}$ for $2 \mathrm{~min}, 40$ cycles of $95^{\circ} \mathrm{C}$ for $15 \mathrm{~s}$ and $60^{\circ} \mathrm{C}$ for $30 \mathrm{~s}$, The expression of glyceraldehyde-3-phosphate dehydrogenase gene (GAPDH) and Eukaryotic elongation factor 1a $(e E F-1 a)$ were used as internal control [79] and the primers of DREB genes are listed in Additional File 6.

\begin{abstract}
Abbreviations
TFs: Transcription factors; DREBs: Dehydration responsive element binding proteins; ABA: Abscisic acid; AP2/ERFBP: APETALA2/ethylene-responsive element-binding protein; AA: The length of protein sequences; MW: The molecular weight; pl: The theoretical isoelectric point; Al: The aliphatic index; GRAVY: The grand average of hydropathicity; II: The instability index; NLS: Nuclear localization signal; Ka: Non-synonymous; Ks: Synonymous; Mya: Million years ago; qRT-PCR: Real time quantitative reverse transcriptionpolymerase chain reaction; FPKM: Fragments Per transcript Kilobase per Million fragments mapped; RNA-seq: RNA Sequencing; HMM: Hidden Markov Model; NJ: Neighbor-joining; RSEM: RNA-Seq by the Expectation-

Maximization; GAPDH: Glyceraldehyde-3-phosphate dehydrogenase; eEF-

1a: Eukaryotic elongation factor 1a
\end{abstract}

\section{Supplementary Information}

The online version contains supplementary material available at https://doi. org/10.1186/s12864-021-07799-5.

Additional file 1 Sequence features of DREBs in S. spontaneum. 
Additional file 2. Alignment of the deducted protein sequences of SSDREBs. The rectangle in black and red represent AP2/ERF domain and motifs (DASW, LWSY, CMIV-1), respectively, the black line represent the NLS sequence.

Additional file $\mathbf{3}$ The segmental or whole-genome duplicated DREB genes in S.spontanuem.

Additional file 4 gRT-PCR verification of SSDREB genes in $S$. spontanenum. a Comparison of qRT-PCR and RNA-seq data of SSDREB genes. $\mathbf{b}$ Correlation coefficient between RNA-seq ( $X$ axis) and qRT-PCR $(Y$ axis) of four SSDREB genes.

Additional file 5 Expression pattern of SSDREB genes in tandemly duplicated regions in S. spontaneum.

Additional file 6. Gene primers used for qRT-PCR analysis.

\section{Authors' contributions}

Z.L. and J.Z. conceived the study and designed the experiments. Z.L., G.W., X.L., Z.W., M.Z., and J.Z. carried out the experiments and analyzed the data. J.Z. and Z.L. wrote the manuscript. All authors read and approved the final paper.

\section{Funding}

The research was supported by grants from program for the National key research and development program (2018YFD1000104), the National Hightech R\&D Program (2013AA100604), the National Natural Science Foundation of China (31201260, 31760413 and 31660420), the Science and Technology Major Project of Guangxi (AA17202025), and the Fujian Provincial Department of Education (No. JA12082).

\section{Availability of data and materials}

All sequence data and phylogeny data during the current study were deposited in the Treebase repository (http://purl.org/phylo/treebase/ phylows/study/TB2:S28119). The genomic data of S. spontaneum was generated in our own laboratory [80] (accession numbers in Genbank: QVOL00000000).

\section{Declarations}

Ethics approval and consent to participate

Not applicable.

\section{Consent for publication}

Not applicable.

\section{Competing interests}

The authors declare that they have no competing interests.

\section{Author details}

${ }^{1}$ Fujian Provincial Key Laboratory of Haixia Applied Plant Systems Biology, College of Agriculture, Fujian Agriculture and Forestry University, Fuzhou 350002, China. ${ }^{2}$ Jiangsu Key Laboratory for Bioresources of Saline Soils, Jiangsu Provincial Key Laboratory of Coastal Wetland Bioresources and Environmental Protection, Jiangsu Synthetic Innovation Center for Coastal Bio-agriculture, Yancheng Teachers University, Yancheng 224051, China. ${ }^{3}$ Sugarcane Research Institute, Guangxi Academy of Agricultural Sciences, Nanning 530007, Guangxi, China. ${ }^{4}$ Provincial Key Laboratory for Developmental Biology and Neurosciences, College of Life Sciences, Fujian Normal University, Fuzhou 350007, China. ${ }^{5}$ Guangxi Key Laboratory of Sugarcane Biology, Guangxi University, Nanning 530004, Guangxi, China.

Received: 12 April 2021 Accepted: 10 June 2021

Published online: 17 June 2021

\section{References}

1. Sakuma Y, Liu Q, Dubouzet JG, Abe H, Shinozaki K, Yamaguchi-Shinozaki K. DNA-binding specificity of the ERF/AP2 domain of Arabidopsis DREBs, transcription factors involved in dehydration- and cold-inducible gene expression. Biochem Biophys Res Commun. 2002;290(3):998-1009. https:// doi.org/10.1006/bbrc.2001.6299.
2. Hasan Khan Z, Kumar B, Dhatterwal P, Mehrotra S, Mehrotra R. Transcriptional regulatory network of cis-regulatory elements (Cres) and transcription factors (TFs) in plants during abiotic stress. Int J Plant Biol Res. 2017:5(2):1064.

3. Yamaguchi-Shinozaki K, Shinozaki K. Organization of cis-acting regulatory elements in osmotic-and cold-stress-responsive promoters. Trends Plant Sci. 2005;10(2):88-94. https://doi.org/10.1016/j.tplants.2004.12.012.

4. Yamaguchi-Shinozaki K, Shinozaki K. Transcriptional regulatory networks in cellular responses and tolerance to dehydration and cold stresses. Annu Rev Plant Biol. 2006;57(1):781-803. https://doi.org/10.1146/annurev.arplant.57.032 905.105444.

5. Yamaguchi-Shinozaki K, Shinozaki K. A novel cis-acting element in an Arabidopsis gene is involved in responsiveness to drought, lowtemperature, or high-salt stress. Plant Cell. 1994;6(2):251-64. https://doi. org/10.1105/tpc.6.2.251.

6. Riechmann JL, Meyerowitz EM. The AP2/EREBP family of plant transcription factors. Biol Chem. 1998;379(6):633-46. https://doi.org/10.1515/bchm.1998.3 79.6.633.

7. Riechmann JL, Heard J, Martin G, Reuber L, Jiang CZ, Keddie J, et al. Arabidopsis transcription factors: genome-wide comparative analysis among eukaryotes. Science (New York, NY). 2000;290(5499):2105-10.

8. Allen MD, Yamasaki K, Ohme-Takagi M, Tateno M, Suzuki M. A novel mode of DNA recognition by a $\beta$-sheet revealed by the solution structure of the GCC-box binding domain in complex with DNA. EMBO J. 1998;17(18):548496. https://doi.org/10.1093/emboj/17.18.5484.

9. Liu S, Wang X, Wang H, Xin H, Yang X, Yan J, et al. Genome-wide analysis of ZmDREB genes and their association with natural variation in drought tolerance at seedling stage of Zea mays L. PLoS Genet. 2013;9(9):e1003790. https://doi.org/10.1371/journal.pgen.1003790.

10. Liu Q, Kasuga M, Sakuma Y, Abe H, Miura S, Yamaguchi-Shinozaki K, et al. Two transcription factors, DREB1 and DREB2, with an EREBP/AP2 DNA binding domain separate two cellular signal transduction pathways in drought-and low-temperature-responsive gene expression, respectively, in Arabidopsis. Plant Cell. 1998;10(8):1391-406. https://doi.org/10.1105/tpc.10 8.1391.

11. Nakashima K, Shinwari ZK, Sakuma Y, Seki M, Miura S, Shinozaki K, et al. Organization and expression of two Arabidopsis DREB2 genes encoding DRE-binding proteins involved in dehydration-and high-salinity-responsive gene expression. Plant Mol Biol. 2000;42(4):657-65. https://doi.org/10.1 023/A:1006321900483.

12. Wei T, Deng K, Liu D, Gao Y, Liu Y, Yang M, et al. Ectopic expression of DREB transcription factor, AtDREB1A, confers tolerance to drought in transgenic Salvia miltiorrhiza. Plant Cell Physiol. 2016;57(8):1593-609. https:/ doi.org/10.1093/pcp/pcw084.

13. Wu H, Li L, Cheng Z, Ge W, Gao J, Li X. Cloning and stress response analysis of the PeDREB2A and PeDREB1A genes in moso bamboo (Phyllostachys edulis). Genet Mol Res. 2015;14(3):10206-23. https://doi.org/10.4238/2015.A ugust.28.4.

14. Qin F, Sakuma Y, Li J, Liu Q, Li YQ, Shinozaki K, et al. Cloning and functional analysis of a novel DREB1/CBF transcription factor involved in coldresponsive gene expression in Zea mays L. Plant Cell Physiol. 2004;45(8): 1042-52. https://doi.org/10.1093/pcp/pch118.

15. Moon S-J, Min MK, Kim J, Kim DY, Yoon IS, Kwon TR, et al. Ectopic expression of OsDREB1G, a member of the OsDREB1 subfamily, confers cold stress tolerance in rice. Front Plant Sci. 2019;10:297. https://doi.org/10.3389/ fpls.2019.00297.

16. Wen W, Xie Z, Yu G, Zhao C, Zhang J, Huang L, et al. Switchgrass PvDREB1C plays opposite roles in plant cold and salt tolerance in transgenic tobacco. Hereditas. 2018;155(1):15. https://doi.org/10.1186/s41065-017-0050-4.

17. Challam C, Ghosh T, Rai M, Tyagi W. Allele mining across DREB1A and DREB1B in diverse rice genotypes suggest a highly conserved pathway inducible by low temperature. J Genet. 2015;94(2):231-8. https://doi.org/10.1 007/s12041-015-0507-z.

18. Sakuma Y, Maruyama K, Qin F, Osakabe Y, Shinozaki K, Yamaguchi-Shinozaki K. Dual function of an Arabidopsis transcription factor DREB2A in waterstress-responsive and heat-stress-responsive gene expression. Proc Natl Acad Sci. 2006;103(49):18822-7. https://doi.org/10.1073/pnas.0605639103.

19. Qin F, Kakimoto M, Sakuma Y, Maruyama K, Osakabe Y, Tran LS, et al. Regulation and functional analysis of ZmDREB2A in response to drought and heat stresses in Zea mays L. Plant J. 2007;50(1):54-69. https://doi.org/1 0.1111/j.1365-313X.2007.03034.X. 
20. Gupta K, Agarwal PK, Reddy M, Jha B. SbDREB2A, an a-2 type DREB transcription factor from extreme halophyte Salicornia brachiata confers abiotic stress tolerance in Escherichia coli. Plant Cell Rep. 2010;29(10):11317. https://doi.org/10.1007/s00299-010-0896-7

21. Gumi AM, Guha PK, Mazumder A, Jayaswal P, Mondal TK. Characterization of OgIDREB2A gene from African rice (Oryza glaberrima), comparative analysis and its transcriptional regulation under salinity stress. 3 Biotech. 2018;8(2):91.

22. Chen H, Liu L, Wang L, Wang S, Cheng X. VrDREB2A, a DREB-binding transcription factor from Vigna radiata, increased drought and high-salt tolerance in transgenic Arabidopsis thaliana. J Plant Res. 2016;129(2):263-73. https://doi.org/10.1007/s10265-015-0773-0.

23. Cong $L$, Zheng HC, Zhang YX, Chai TY. Arabidopsis DREB1A confers high salinity tolerance and regulates the expression of GA dioxygenases in tobacco. Plant Sci. 2008;174(2):156-64. https://doi.org/10.1016/j.plantsci.2 007.11.002.

24. Zhang XX, Tang YJ, Ma QB, Yang CY, Mu YH, Suo HC, et al. OsDREB2A, a rice transcription factor, significantly affects salt tolerance in transgenic soybean. PLoS One. 2013;8(12):e83011. https://doi.org/10.1371/journal.pone.0083011.

25. Wei T, Deng K, Gao Y, Liu Y, Yang M, Zhang L, et al. Arabidopsis DREB1B in transgenic Salvia miltiorrhiza increased tolerance to drought stress without stunting growth. Plant Physiol Biochem. 2016;104:17-28. https://doi.org/10.1 016/j.plaphy.2016.03.003.

26. El-Esawi MA, Alayafi AA. Overexpression of StDREB2 transcription factor enhances drought stress tolerance in cotton (Gossypium barbadense L.). Genes. 2019;10(2):142.

27. Roach B. Nobilisation of sugarcane. Proc Int Soc Sugar Cane Technol. 1972; 1972:206-16.

28. Reis RR, da Cunha BA, Martins PK, Martins MT, Alekcevetch JC, Chalfun A Jr, et al. Induced over-expression of AtDREB2A CA improves drought tolerance in sugarcane. Plant Sci. 2014;221-222:59-68. https://doi.org/10.1016/j.pla ntsci.2014.02.003.

29. Augustine SM, Ashwin Narayan J, Syamaladevi DP, Appunu C, Chakravarthi $M$, Ravichandran $V$, et al. Overexpression of EaDREB2 and pyramiding of EaDREB2 with the pea DNA helicase gene (PDH45) enhance drought and salinity tolerance in sugarcane (Saccharum spp. hybrid). Plant Cell Rep. 2015; 34(2):247-63. https://doi.org/10.1007/s00299-014-1704-6.

30. Huang X, Song X, Chen R, Zhang B, Li C, Liang Y, et al. Genome-wide analysis of the DREB subfamily in Saccharum spontaneum reveals their functional divergence during cold and drought stresses. Front Genet. 2019; 10:1326.

31. Solovyev V, Kosarev P, Seledsov I, Vorobyev D. Automatic annotation of eukaryotic genes, pseudogenes and promoters. Genome Biol. 2006;7(Suppl 1):S10.11-2.

32. Holub EB. The arms race is ancient history in Arabidopsis, the wildflower. Nat Rev Genet. 2001;2(7):516-27. https://doi.org/10.1038/35080508.

33. Zhang J, Zhang Q, Li L, Tang H, Zhang Q, Chen Y, et al. Recent polyploidization events in three Saccharum founding species. Plant Biotechnol J. 2019;17(1):264-74. https://doi.org/10.1111/pbi.12962.

34. Li P, Ponnala L, Gandotra N, Wang L, Si Y, Tausta SL, et al. The developmental dynamics of the maize leaf transcriptome. Nat Genet. 2010; 42(12):1060-7. https://doi.org/10.1038/ng.703.

35. Yang H, Wang T, Yu X, Yang Y, Wang C, Yang Q, et al. Enhanced sugar accumulation and regulated plant hormone signalling genes contribute to cold tolerance in hypoploid Saccharum spontaneum. BMC Genomics. 2020; 21(1):507. https://doi.org/10.1186/s12864-020-06917-z.

36. Lee SC, Lim MH, Yu JG, Park BS, Yang TJ. Genome-wide characterization of the CBF/DREB1 gene family in Brassica rapa. Plant Physiol Biochem. 2012;61: 142-52. https://doi.org/10.1016/j.plaphy.2012.09.016.

37. Matsukura S, Mizoi J, Yoshida T, Todaka D, Ito Y, Maruyama K, et al. Comprehensive analysis of rice DREB2-type genes that encode transcription factors involved in the expression of abiotic stress-responsive genes. Mol Gen Genomics. 2010;283(2):185-96. https://doi.org/10.1007/s00438-0090506-y.

38. Mao D, Chen C. Colinearity and similar expression pattern of rice DREB1s reveal their functional conservation in the cold-responsive pathway. PLoS One. 2012;7(10):e47275. https://doi.org/10.1371/journal. pone.0047275.

39. Skinner JS, von Zitzewitz J, Szucs P, Marquez-Cedillo L, Filichkin T, Amundsen $K$, et al. Structural, functional, and phylogenetic characterization of a large CBF gene family in barley. Plant Mol Biol. 2005;59(4):533-51. https://doi.org/10.1007/s11103-005-2498-2.
40. Akbudak MA, Filiz E, Kontbay K. DREB2 (dehydration-responsive elementbinding protein 2) type transcription factor in sorghum (Sorghum bicolor): genome-wide identification, characterization and expression profiles under cadmium and salt stresses. 3 Biotech. 2018;8(10):426.

41. Agarwal PK, Agarwal P, Reddy MK, Sopory SK. Role of DREB transcription factors in abiotic and biotic stress tolerance in plants. Plant Cell Rep. 2006; 25(12):1263-74. https://doi.org/10.1007/s00299-006-0204-8.

42. Lakhssassi N, Liu S, Bekal S, Zhou Z, Colantonio V, Lambert K, et al. Characterization of the soluble NSF attachment protein gene family identifies two members involved in additive resistance to a plant pathogen. Sci Rep. 2017;7(1):45226. https://doi.org/10.1038/srep45226.

43. Perez-Perez JM, Esteve-Bruna D, Gonzalez-Bayon R, Kangasjarvi S, Caldana C, Hannah MA, et al. Functional redundancy and divergence within the Arabidopsis RETICULATA-RELATED gene family. Plant Physiol. 2013;162(2): 589-603. https://doi.org/10.1104/pp.113.217323.

44. Lakhssassi N, Doblas VG, Rosado A, del Valle AE, Pose D, Jimenez AJ, et al. The Arabidopsis tetratricopeptide thioredoxin-like gene family is required for osmotic stress tolerance and male sporogenesis. Plant Physiol. 2012; 158(3):1252-66. https://doi.org/10.1104/pp.111.188920.

45. Akhtar M, Jaiswal A, Taj G, Jaiswal J, Qureshi M, Singh N. DREB1/CBF transcription factors: their structure, function and role in abiotic stress tolerance in plants. J Genet. 2012;91(3):385-95. https://doi.org/10.1007/s12 041-012-0201-3.

46. Dubouzet JG, Sakuma Y, Ito Y, Kasuga M, Dubouzet EG, Miura S, et al. OsDREB genes in rice, Oryza sativa L., encode transcription activators that function in drought-, high-salt-and cold-responsive gene expression. Plant J. 2003;33(4):751-63. https://doi.org/10.1046/j.1365-313X.2003.01661.X.

47. Jaglo KR, Kleff S, Amundsen KL, Zhang X, Haake V, Zhang JZ, et al. Components of the Arabidopsis C-repeat/dehydration-responsive element binding factor cold-response pathway are conserved inbrassica napus and other plant species. Plant Physiol. 2001;127(3):910-7. https://doi.org/10.1104/ pp.010548.

48. Zhou ML, Ma JT, Pang JF, Zhang ZL, Tang YX, Wu YM. Regulation of plant stress response by dehydration responsive element binding (DREB) transcription factors. Afr J Biotechnol. 2010;9(54):9255-69.

49. Herath V. Small family, big impact: in silico analysis of DREB2 transcription factor family in rice. Comput Biol Chem. 2016;65:128-39. https://doi.org/10.1 016/j.compbiolchem.2016.10.012.

50. Söderman EM, Brocard IM, Lynch TJ, Finkelstein RR. Regulation and function of the Arabidopsis ABA-insensitive4 gene in seed and abscisic acid response signaling networks. Plant Physiol. 2000;124(4):1752-65. https://doi.org/10.11 04/pp.124.4.1752.

51. Shkolnik-Inbar D, Bar-Zvi D. ABI4 mediates abscisic acid and cytokinin inhibition of lateral root formation by reducing polar auxin transport in Arabidopsis. Plant Cell. 2010;22(11):3560-73. https://doi.org/10.1105/tpc.110. 074641.

52. Niehrs C, Pollet N. Synexpression groups in eukaryotes. Nature. 1999; 402(6761):483-7. https://doi.org/10.1038/990025.

53. Ni Z, Kim E-D, Ha M, Lackey E, Liu J, Zhang Y, et al. Altered circadian rhythms regulate growth vigour in hybrids and allopolyploids. Nature. 2009; 457(7227):327-31. https://doi.org/10.1038/nature07523.

54. Green RM, Tingay S, Wang Z-Y, Tobin EM. Circadian rhythms confer a higher level of fitness to Arabidopsis plants. Plant Physiol. 2002;129(2):576-84. https://doi.org/10.1104/pp.004374.

55. Michael TP, Salome PA, Hannah JY, Spencer TR, Sharp EL, McPeek MA, et al. Enhanced fitness conferred by naturally occurring variation in the circadian clock. Science. 2003;302(5647):1049-53. https://doi.org/10.1126/science.1082 971.

56. Dodd AN, Salathia N, Hall A, Kévei E, Tóth R, Nagy F, et al. Plant circadian clocks increase photosynthesis, growth, survival, and competitive advantage. Science. 2005;309(5734):630-3. https://doi.org/10.1126/ science.1115581.

57. Giuliano G, Hoffman N, Ko K, Scolnik P, Cashmore A. A light-entrained circadian clock controls transcription of several plant genes. EMBO J. 1988; 7(12):3635-42. https://doi.org/10.1002/j.1460-2075.1988.tb03244.X.

58. Xu Z, Ma J, Qu C, Hu Y, Hao B, Sun Y, et al. Identification and expression analyses of the alanine aminotransferase (AlaAT) gene family in poplar seedlings. Sci Rep. 2017;7(1):1-13.

59. Bihani $P$, Char B, Bhargava S. Transgenic expression of sorghum DREB2 in rice improves tolerance and yield under water limitation. J Agric Sci. 2011; 149(1):95-101. https://doi.org/10.1017/S0021859610000742. 
60. Zhang J, Arro J, Chen Y, Ming R. Haplotype analysis of sucrose synthase gene family in three Saccharum species. BMC Genomics. 2013;14(1):314. https://doi.org/10.1186/1471-2164-14-314.

61. Irvine J. Saccharum species as horticultural classes. Theor Appl Genet. 1999; 98(2):186-94. https://doi.org/10.1007/s001220051057.

62. Li Z, Hua X, Zhong W, Yuan Y, Wang Y, Wang Z, et al. Genome-wide identification and expression profile analysis of WRKY family genes in the autopolyploid Saccharum spontaneum. Plant Cell Physiol. 2020;61(3):616-30. https://doi.org/10.1093/pcp/pcz227.

63. Zhang Q, Hu W, Zhu F, Wang L, Yu Q, Ming R, et al. Structure, phylogeny, allelic haplotypes and expression of sucrose transporter gene families in Saccharum. BMC Genomics. 2016;17(1):88. https://doi.org/10.1186/s12864-01 6-2419-6.

64. Hu W, Hua X, Zhang Q, Wang J, Shen Q, Zhang X, et al. New insights into the evolution and functional divergence of the SWEET family in Saccharum based on comparative genomics. BMC Plant Biol. 2018;18(1):270. https://doi. org/10.1186/s12870-018-1495-y.

65. Ming R, VanBuren R, Wai CM, Tang H, Schatz MC, Bowers JE, et al. The pineapple genome and the evolution of CAM photosynthesis. Nat Genet. 2015;47(12):1435-42. https://doi.org/10.1038/ng.3435.

66. El-Gebali S, Mistry J, Bateman A, Eddy SR, Luciani A, Potter SC, et al. The Pfam protein families database in 2019. Nucleic Acids Res. 2018;47(D1): D427-32.

67. Nakano T, Suzuki K, Fujimura T, Shinshi H. Genome-wide analysis of the ERF gene family in Arabidopsis and rice. Plant Physiol. 2006;140(2):411-32. https://doi.org/10.1104/pp.105.073783.

68. Hu B, Jin J, Guo AY, Zhang H, Luo J, Gao G. GSDS 2.0: an upgraded gene feature visualization server. Bioinformatics. 2014;31(8):1296-7. https://doi. org/10.1093/bioinformatics/btu817.

69. Chen C, Chen H, Zhang Y, Thomas HR, Frank MH, He Y, Xia R. TBtools: An Integrative Toolkit Developed for Interactive Analyses of Big Biological Data. Mol Plant. 2020;13(8):1194-202.

70. Easy_KaKs. https://github.com/tangerzhang/FAFU-cgb/tree/master/easy_Ka Ks. Accessed 1 Oct 2018.

71. Lynch M, Conery JS. The evolutionary fate and consequences of duplicate genes. Science. 2000;290(5494):1151-5. https://doi.org/10.1126/science.290 5494.1151

72. Kumar S, Stecher G, Tamura K. MEGA7: molecular evolutionary genetics analysis version 7.0 for bigger datasets. Mol Biol Evol. 2016;33(7):1870-4. https://doi.org/10.1093/molbev/msw054.

73. Felsenstein J. Confidence limits on phylogenies: an approach using the bootstrap. Evolution. 1985;39(4):783-91. https://doi.org/10.1111/j.1558-5646.1 985.tb00420.x.

74. Saitou N, Nei M. The neighbor-joining method: a new method for reconstructing phylogenetic trees. Mol Biol Evol. 1987;4(4):406-25. https:// doi.org/10.1093/oxfordjournals.molbev.a040454.

75. Letunic I, Bork P. Interactive Tree Of Life (iTOL) v5: an online tool for phylogenetic tree display and annotation. Nucleic Acids Res. 2021;gkab301. https://doi.org/10.1093/nar/gkab301.

76. Wang Y, Tang H, DeBarry JD, Tan X, Li J, Wang X, et al. MCScanX: a toolkit for detection and evolutionary analysis of gene synteny and collinearity. Nucleic Acids Res. 2012;40(7):e49. https://doi.org/10.1093/nar/gkr1293.

77. Bolger AM, Lohse M, Usadel B. Trimmomatic: a flexible trimmer for Illumina sequence data. Bioinformatics. 2014;30(15):2114-20. https://doi.org/10.1093/ bioinformatics/btu170

78. Haas BJ, Papanicolaou A, Yassour M, Grabherr M, Blood PD, Bowden J, et al. De novo transcript sequence reconstruction from RNA-seq using the trinity platform for reference generation and analysis. Nat Protoc. 2013;8(8):1494512. https://doi.org/10.1038/nprot.2013.084.

79. Ling H, Wu Q, Guo J, Xu L, Que Y. Comprehensive selection of reference genes for gene expression normalization in sugarcane by real time quantitative RT-PCR. PLoS One. 2014;9(5):e97469. https://doi.org/10.1371/ journal.pone.0097469.

80. Zhang J, Zhang X, Tang H, Zhang Q, Hua X, Ma X, et al. Allele-defined genome of the autopolyploid sugarcane Saccharum spontaneum L. Nat Genet. 2018;50(11):1565-73. https://doi.org/10.1038/s41588-018-0237-2.

\section{Publisher's Note}

Springer Nature remains neutral with regard to jurisdictional claims in published maps and institutional affiliations.

\section{Ready to submit your research? Choose BMC and benefit from:}

- fast, convenient online submission

- thorough peer review by experienced researchers in your field

- rapid publication on acceptance

- support for research data, including large and complex data types

- gold Open Access which fosters wider collaboration and increased citations

- maximum visibility for your research: over $100 \mathrm{M}$ website views per year

At BMC, research is always in progress.

Learn more biomedcentral.com/submissions 